\title{
Experimental and numerical investigation on the impact of vacuum level on effective thermal conductivity and microstructure of carbon fibre reinforced epoxy composites manufactured by vacuum bag method
}

\author{
Michat Kubisi ${ }^{1, *}$, Mirostaw Seredyński ${ }^{1}$, Łukasz Cieślikiewicz ${ }^{1}$, Tomasz Wiśniewski ${ }^{1}$, and Anna Boczkowska ${ }^{2}$ \\ ${ }^{1}$ Institute of Heat Engineering, Faculty of Power and Aeronautical Engineering, Warsaw University of Technology, 21/25 Nowowiejska \\ Str., 00-665 Warsaw, Poland \\ ${ }^{2}$ Faculty of Materials Science and Engineering, Warsaw University of Technology, \\ 02-507 Warsaw, Poland
}

\begin{abstract}
The physical properties of epoxy based carbon reinforced composites are highly anisotropic due to their directional structure and dependent on the manufacturing process parameters. Thermal conductivity was found to be dependent on the void volume fractions, which appear as a result of the insufficient vacuum level. In the proposed paper the multi-scale computational model of heat transfer across the carbon fiber-epoxy resin composite is proposed. The meso-scale effective thermal conductivities are determined with analytical formulae for isotropic and anisotropic media, the latter takes into account thermal resistance at the interface of fibres and epoxy resin. Proposed model is utilized to determine the effective thermal conductivity in the direction perpendicular to plies of composite. The influence of void fractions and the thickness of the composite on the effective thermal conductivity is investigated. The numerical outcomes underestimate the real variation in conductivity, which can be caused by change in carbon volume fraction of samples manufactured at different vacuum levels which was not considered in numerical computation.
\end{abstract}

\section{Introduction}

Epoxy based carbon reinforced composites, especially those with long fibers are well known for their high strength to density ratio as well as strong anisotropy of their properties both mechanical and physical [1,2]. It is a result of layered structure and fibers alignment e.g. unidirectional, braided or fabric. On the other hand, microstructure quality is the second factor that is listed to have great impact on effective thermal conductivity. Void volume fraction and fiber volume fraction are two parameters that in minority affects overall properties of composites [3]. They could vary amongst different manufacturing methods and be altered by manufacturing process parameters e.g. vacuum level in the case of methods based on a vacuum bag.

Effective thermal conductivity of composites became significant factor as their structures became designing to work in environment of higher temperature gradients e.g. engine casing, battery casing, composite tooling [4] as well as wing shields in aeroplane which surface heats up to more than $100^{\circ} \mathrm{C}$ during the flight. According to complex alignment of fibers in carbon fabrics braids infiltrated with epoxy resin their effective thermal conductivity are not obvious to predict.

Classical theoretical models: effective medium approximation methods $[5,6,7,9,10]$ or based on micromechanics $[11,12,13,14]$ are well known and very simple, and quick to apply. Nevertheless, their scope of application is limited to simplified cases due to composites parameters e.g. particle arrangement, their shape, restricted volume fractions or considering of interfacial contact resistance.

Effective thermal conductivity could be computed also based on simulation e.g. FEM, FDM, Element Free Galerkin, Lattice Boltzman Method, off-lattice Monte Carlo as well as Molecular Dynamics [15]. The great advantage of simulation approach is their ability to accurate characterization of details of composite microstructure at different scales: micro-scale, meso-scale and macro-scale. However, simulation of multiscaled microstructure requires to determine a large representative elementary volume to evaluate effective thermal conductivity accurately. Hence great computing power demand is the bottleneck of these methods especially if dimensions of microstructure elements vary in orders of magnitude. Wherefore multiscale modeling based of

\footnotetext{
Corresponding author: mkubis@itc.pw.edu.pl
} 
integrating of above-mentioned methods could provide efficient solution for that inconvenience.

As the input to the model several micro-scale properties are needed. Among them are thermal conductivity of a single carbon fiber in parallel and radial directions, and thermal conductance at the interface of fibre and matrix material. In both cases literature review indicates that there is a wide range of values reported by many authors. Thermal conductivity in parallel direction can be as high as 4.4-75 $\mathrm{W} /(\mathrm{m} \cdot \mathrm{K})$ for PAN-based carbon fibres $[16,17,18]$ and $0.5-25$ $\mathrm{W} /(\mathrm{m} \cdot \mathrm{K})$ in radial direction $[19,20]$.

Values of thermal conductance showed in literature, or its reciprocial, the interface resistance, $R_{k}$, cover wide range. Hua et al. [20] coupled the LFA experimental analysis and simplified numerical simulations to predict effective thermal conductivity along fibers and interface resistance. The procedure of tuning of both parameters was based on finding the best correlation between experimental and numerically predicted signal curve. The considered thermal resistances were in the range $10^{-6}$ and $10^{-4} \mathrm{~m}^{2} \mathrm{~K} / \mathrm{W}$. The best fitted value determined was $10^{-5} \mathrm{~m}^{2} \mathrm{~K} / \mathrm{W}$.

Bui et al. [21] calculated the effective thermal conductivity of the carbon nanotube-polystyrene composite. They estimated the interfacial resistance by tuning the results given with Molecular Dynamics simulations and experimental findings. The calculated resistance between carbon nanotube and polymer was $2.21 \cdot 10^{-8} \mathrm{~m}^{2} \mathrm{~K} / \mathrm{W}$.

The objective of this paper was to investigate the impact of manufacturing parameter on composite structure. Therefore three types of composites were manufactured with vacuum assisted resin infusion method at three different levels of vacuum. Then their physical properties were measured: thermal diffusivity $a$ (LFA 447 Nanoflash, Netzsch) and specific heat $c_{p}$ (DSC 404 Pegasus, Netzsch). Density $\rho$ was calculated based on geometry and mass measurement. Thermal conductivity in through the thickness direction was determined according to formula: $k=a \cdot c_{p} \cdot \rho$. Microstructures of composites were investigated with scanning electron microscopy. Quantitive 2D image analysis technique was utilized to determine void volume fractions (Micrometer program [22, 23]). Based on the same images, geometry of the composite structure was developed and model of medium approximation integrated with numerical simulation were applied for effective thermal conductivity calculation. Both results were compared and discussed.

\section{Structure of the composite}

Six layers of plain fabric $\left(200 \mathrm{~g} / \mathrm{m}^{2}\right)$ made of carbon fibers were infiltrated with Axson epoxy system consist of a resin Epolam 2031 and a hardener Epolam 2031. SEM image shows exemplary cross section of composite where yarns/tows are interlaced and positioned perpendicular to each other and denoted as numbers 1 and 2 (Fig.1). Single carbon fibers within yarns/tows are the brithest elements of image infiltrated with resin that is pictured herein as a darker shades of gray and the black areas are voids.

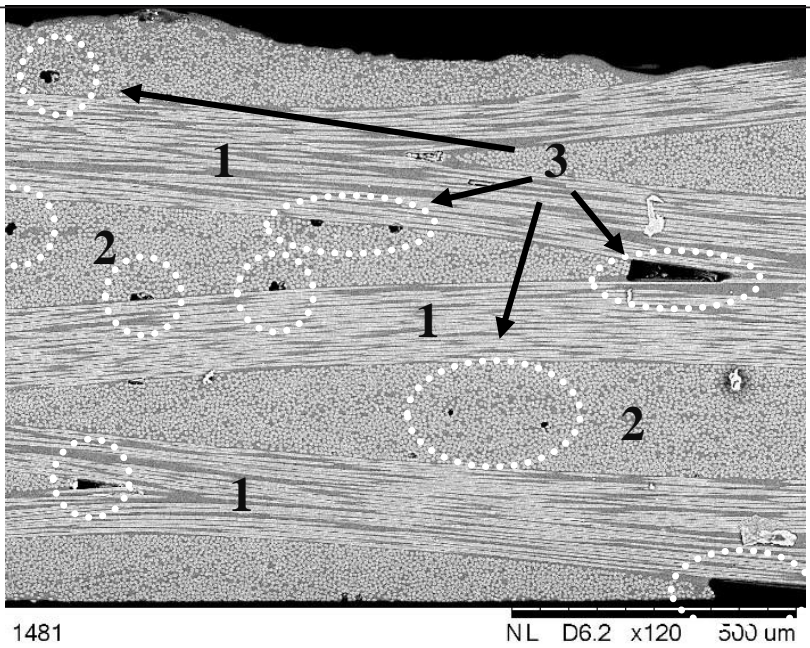

Fig. 1. Exemplary SEM image of composite microstructure. 1fiber tows transversal to normal of crossection, 2-fibre tows parallel to normal of crosssection, 3-voids.

Three different types of samples were made at different vacuum levels applied during manufacturing process. Value of vacuum levels presented in table 1 referred to atmospheric pressure. Detailed observations of bunches of carbon fibers, done with a scanning electron microscope in the cross-sections normal and parallel to fibers, revealed very good penetration of bunches with epoxy resin. Although small amount of voids appeared as a result of entrapped air during infiltration process. Each type of manufactured samples was investigated.

Experimental results of effective thermal conductivity in through the thickness direction were shown in table 1, as well as void volume fraction and manufacturing parameters.

Table 1. Experimental results of effective thermal conductivity and void volume fraction at various manufacturing conditions.

\begin{tabular}{|c|c|c|}
\hline $\begin{array}{c}\text { Vacuum level below } \\
\text { atmospheric pressure } \\
{[\mathrm{kPa}]}\end{array}$ & $\begin{array}{c}\text { Volume fraction } \\
\text { of voids }[\%]\end{array}$ & $\begin{array}{c}\text { Effective } \\
\text { thermal } \\
\text { conductivity } \\
{[\mathrm{W} /(\mathrm{m} \cdot \mathrm{K})]}\end{array}$ \\
\hline-90 & 1,6 & 0.54 \\
\hline-60 & 2,5 & 0.50 \\
\hline-30 & 3,5 & 0.45 \\
\hline
\end{tabular}

\section{Analysis and modelling}

\subsection{Geometry}

Based on detailed measurements of the composite geometry, its threedimensional model is developed. The composite has the multi-scale structure. At the meso-scale the discernible components are yarn, warp and the matrix, epoxy resin. At the micro-scale, both the yarn and warp are bunches of 
parallel carbon fibres infiltrated with the epoxy resin. For the sake of analysis simplification both yarn and warp are treated as continuous with the same structure and properties..

The simplified geometry of warp and yarn (Fig. 2) reflects the real one observed with the scanning microscope (Fig. 1). The computational domain is the cuboid which dimensions are $L_{x}, L_{y}$ and $L_{z}$, measured in directions along $\mathrm{X}, \mathrm{Y}$ and $\mathrm{Z}$ axes of the Cartesian coordinate system, respectively. At the plane perpendicular to fibers the shape of cross-section of the bunch is an ellipse with diameters $d$ and $D$, the former is measured in the $\mathrm{Z}$ direction and the latter in the $\mathrm{X}$ or $\mathrm{Y}$ direction, dependent on the configuration (Fig. 2b). Measured diameters $d$ and $D$ were equal to $0.2 \mathrm{~mm}$ and $2.373 \mathrm{~mm}$. Volume representing the single bunch is formed by swapping the ellipse along a path having the shape of the cosine function, which period is equal to doubled length of the domain, $2 L_{x}$ or $2 L_{y}$, dependent on its orientation. The amplitude is equal to $0.22 \mathrm{~mm}$. For considered case the half distance of the yarn/warp structure period, namely $L_{x}$ or $L_{y}$, is equal to $2.5 \mathrm{~mm}$. The total height of the composite, $L_{z}$, is equal to $0.6 \mathrm{~mm}$. Three layers of weaves are taken into account (Fig. 2). The distance between the horizontal axes of two corresponding layers of bunches is equal to $L_{z}=0.6 \mathrm{~mm}$. The intermediate layer is positioned in the midway between them and it is additionally shifted in the $\mathrm{X}$ and $\mathrm{Y}$ directions by the half of the cavity width, namely $L_{x} / 2$ or $L_{y} / 2$.

The volumetric fraction of all bunches in the whole domain is $94 \%$.

a)

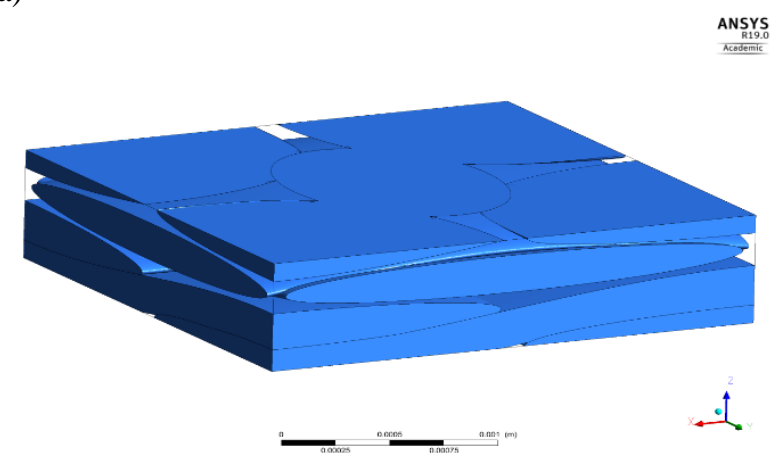

b)

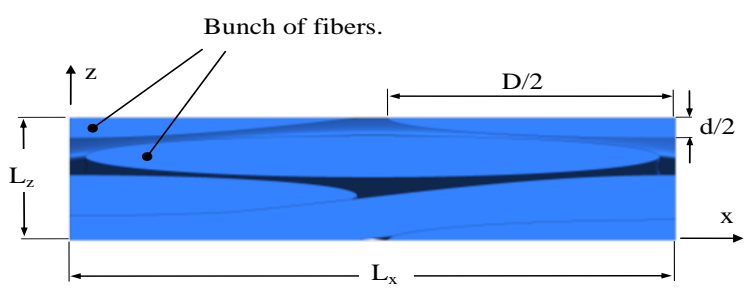

Fig. 2. Isometric view of the considered part of the composite (a) and the side view with dimensions of the macro-structures (b). Bunches of fibers are denoted with pale blue color and the filler, not marked here, fills the rest of the box.

\subsection{Mathematical model}

In the domain presented in Fig. 1, the stationary heat conduction equation is solved

$$
\nabla(k \nabla T)=0
$$

where the thermal conductivity, $k$, is evaluated in each mesoscopic zone (yarn, warp or epoxy resin) of the domain. Due to the non-uniform structure of all zones, their effective mesoscopic thermal conductivities are calculated with the averaging formula. The thermal resistance at the interface of various materials, i.e. fibers and epoxy, is taken into account. The matrix material, namely epoxy resin, has also nonuniform structure, due to presence of entrapped bubbles of air. The averaged thermal conductivity of the matrix material is determined on the assumption, the entrapped gas is uniformly distributed in the epoxy resin, present outside and inside bunches of fibers.

The effective thermal conductivity of the matrix material, $\mathrm{km}$, which is considered as the uniform mixture of the entrapped gas (voids) and epoxy resin, is constant and isotropic. It is derived with the Maxwell model [24],

$$
k_{m}=k_{e}\left[\frac{k_{v}+2 k_{e}+2 \phi_{v}\left(k_{v}-k_{e}\right)}{k_{v}+2 k_{e}-\phi_{v}\left(k_{v}-k_{e}\right)}\right]
$$

as the function of thermal conductivities of epoxy, $k_{e}$, and voids, $k_{v}$, and volume fraction of voids, $\phi_{v}$.

The effective thermal conductivity of bunch of carbon fibers filled with epoxy is highly anisotropic and can be expressed as the diagonal tensor according to its main directions, parallel or perpendicular to fibers. For the former orientation the average thermal conductivity is given with the mixture law

$$
k_{b e f f}^{p}=\phi_{f} k_{f}^{p}+\left(1-\phi_{f}\right) k_{m}
$$

where $\phi_{f}$ is the volume fraction of fibers in the yarn or warp. Based on the analysis of photos of the microstructure it is estimated to be equal to 0.55 , so the volume fraction of carbon fibers in the whole composite is $51.7 \%$. The thermal conductivity of the matrix material, $k_{m}$, takes into account presence of voids in between carbon fibers (eq. 2).

The effective thermal conductivity of the composite, where the thermal resistance, related to imperfect contact of particles and matrix material or the Kapitza resistance [25], should not be neglected, the Hasselmann-Johnson relation can be used. This was originally derived for spherical inclusions and next was generalized for cases involving sets of parallel fibers immersed in the matrix material [7],

$$
k_{b e f f}^{n}=k_{m}\left[\frac{\left(\alpha_{1}-\alpha_{2}-1\right) \phi_{f}+\left(1+\alpha_{1}+\alpha_{2}\right)}{\left(1-\alpha_{1}+\alpha_{2}\right) \phi_{f}+\left(1+\alpha_{1}+\alpha_{2}\right)}\right]
$$

where

$\alpha_{1}=\frac{k_{f}^{n}}{k_{m}}$ and $\alpha_{2}=\frac{k_{f}^{n}}{a h_{C}}$. 
where $\mathrm{a}$ is the radius of fiber and hc is the effective thermal conductance, reciprocial of the thermal resistance, $R_{k}$. Based on the analysis of microstructure of the composite crossection perpendicular to warp, the average radius of single carbon fiber was estimated to be $3.5 \mu \mathrm{m}$.

Both yarn and warp are interlaced and crimped, so fibers are not perfectly straight, parallel to axes of the Cartesian coordinate system. For general case the components of the thermal conductivity tensor must be projected orthogonally on the directions of the fixed Cartesian coordinate system, related to orientation of the composite. To fix the idea the bunch of fibers shown in Fig. $2 b$, parallel to $X$ axis is considered. The components of thermal conductivity in the global coordinate system are functions of squared sine and cosine functions of angle of fibers inclination, $\theta(x)$, with respect to the $\mathrm{X}$ axis [26]. For considered geometry and arrangement of bunches, the maximum value for $\theta$ is estimated to be approximately $6.28^{\circ}$, so variation in the thermal conductivity with changes of the inclination angle of bunch of fibers can be neglected in this preliminary study, and the diagonal components of the tensor of conductivity are simplified to the form

$$
\begin{aligned}
& k_{b, x} \approx k_{b, e f f}^{p} \\
& k_{b, y} \approx k_{b, e f f}^{n} \\
& k_{b, z} \approx k_{b, e f f}^{n}
\end{aligned}
$$

The thermal conductivities of all substances are listed in the table 2 .

Table 2. Material properties of the components of the composite.

\begin{tabular}{|l|c|c|}
\hline & Units & Value \\
\hline $\begin{array}{l}\text { Thermal conductivity of epoxy } \\
\text { resin, } k_{e}\end{array}$ & $\mathrm{~W} /(\mathrm{m} \cdot \mathrm{K})$ & $\begin{array}{l}0.19 \\
{[26]}\end{array}$ \\
\hline $\begin{array}{l}\text { Thermal conductivity of voids, } \\
k_{v}\end{array}$ & $\mathrm{~W} /(\mathrm{m} \cdot \mathrm{K})$ & 0.01 \\
\hline $\begin{array}{l}\text { Thermal conductivity of the } \\
\text { fiber, parallel to axis, } k_{f}\end{array}$ & $\mathrm{~W} /(\mathrm{m} \cdot \mathrm{K})$ & $30[16]$ \\
\hline $\begin{array}{l}\text { Thermal conductivity of the } \\
\text { fiber, perpendicular to axis, } k_{f}\end{array}$ & $\mathrm{~W} /(\mathrm{m} \cdot \mathrm{K})$ & $25[27]$ \\
\hline
\end{tabular}

\subsection{Mesh}

The domain presented in Fig. 2a was spatially discretized with control volume grid refined close to curved surfaces. The maximum size of the control volume (c.v.) face is $0.02 \mathrm{~mm}$, total number of control volumes is $2.31 \mathrm{mln}$. The general quality of mesh was good, but due to geometry constraints, some highly skewed control volumes were created close to edges common for bunches and matrix material. Views of the meshed cross-section of the composite, meshed surface of bunches are shown in Fig. 3.
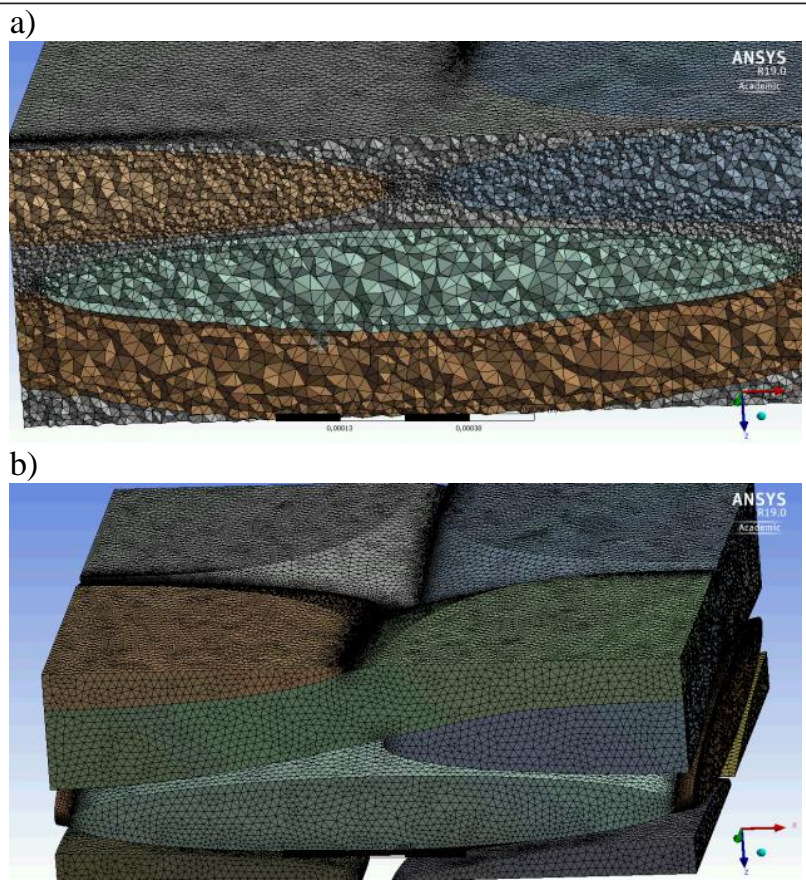

Fig. 3. View of the meshed cross-section of the domain (a) and meshed surface of bunches (b).

The isothermal conditions were imposed at two opposite external surfaces $Z=$ const, namely temperature $300 \mathrm{~K}$ on the $Z$ min and $301 \mathrm{~K}$ on $Z_{\max }$. At other external surfaces the adiabatic condition was imposed. The stationary heat conduction problem was solved with the ANSYS Fluent software. Gradients of temperature were derived with the Least Squares Cell Based scheme and warped-face gradient correction was utilized to avoid nonphysical oscillations. The underrelaxation coefficient was set to 0.8 .

\section{Results and discission}

Five samples of each type of composites were prepared for measurement of their physical properties. Hence experimental values of effective thermal conductivity showed in table 1 were determined as an average results of five samples. The values vary between $0.45-0.54[\mathrm{~W} /(\mathrm{m} \cdot \mathrm{K}])$ along with decreasing vacuum level below atmospheric ($30 \mathrm{kPa}$ to $-90 \mathrm{kPa})$. Void volume fractions were obtained according to quantitative image analysis technique on the basis of analysis of 30 SEM images of 5 different samples for each composite type.

At the first step of setting simulation conditions, the thermal resistance at the surface of carbon fibers was approximated. This was estimated for the volume fraction of entrapped gas in the epoxy resin, equal to $1.6 \%$. The calculated with the Maxwell formula (eq. 2) effective thermal conductivity of epoxy resin with entrapped gas is 0.186 $\mathrm{W} /(\mathrm{m} \cdot \mathrm{K})$.

The thermal conductivity of the bunch, predicted in the normal direction to fibers with the H-J formula (eg. 4), as a function of the radial thermal conductivity, $k_{f}^{n}$ and interfacial 
thermal resistance, $R_{k}$, is presented in Fig. 4. It is evident that the interfacial resistance has considerable impact on the effective thermal conductivity. The red line represents the value of the experimentally determined through-thickness thermal conductivity of the composite. The total effective conductivity of the composite is an average value of conductivities of the epoxy resin, $\mathrm{km}$, and bunch of fibers, in normal direction to fibers, $k_{b, e f f}^{n}$. Since $k_{m}$ is lower than $k_{b, e f f}^{n}$, the latter must be higher than the measured thermal conductivity, so allowable values of the $k_{b, e f f}^{n}$ there are on the left side of the red line in Fig. 4.

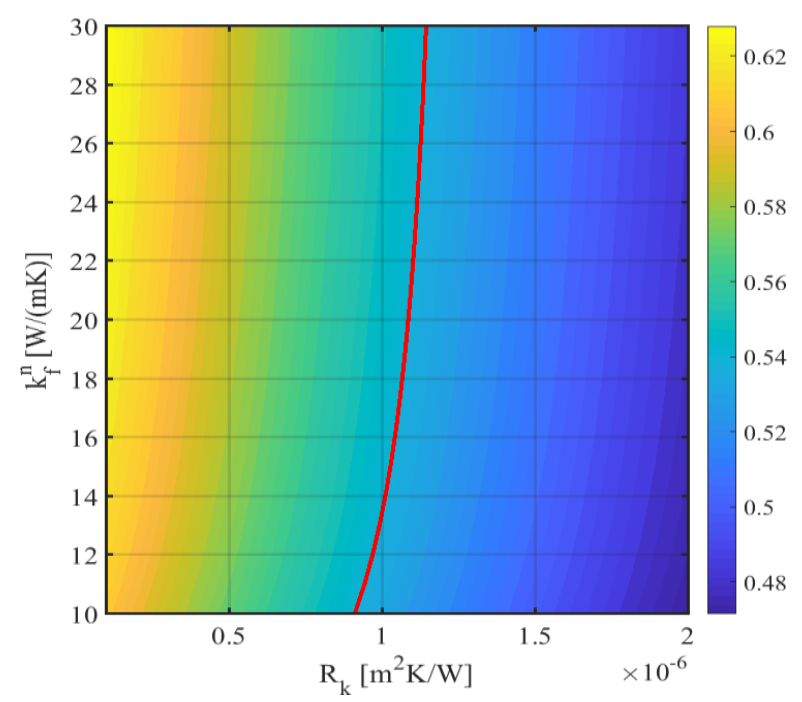

Fig. 4. Effective thermal conductivity of the bunch of fibers immersed in epoxy resin, perpendicular to fibers, predicted with the H-J model (eq. 4). The isoline of conductivity equal to 0.54 $\mathrm{W} /(\mathrm{m} \cdot \mathrm{K})$ is shown with red line.

A series of simulations was performed for the set of thermal interfacial resistances, in the range $10-8$ to $10-6$ $\mathrm{m}^{2} \mathrm{~K} / \mathrm{W}$ and constant radial thermal conductivity of fibers, $k_{f}^{n}$, equal to $25 \mathrm{~W} /(\mathrm{m} \cdot \mathrm{K})$. As a result, the heat flux density, $q$, was determined on two opposite surfaces, Zmin and Zmax, where temperature boundary conditions were imposed. The effective thermal conductivities were determined with the formula:

$$
k_{z, e f f}=q \frac{L_{Z}}{\Delta T}
$$

where $\Delta T$ is the imposed temperature difference, equal to $1 \mathrm{~K}$. Calculated through-thickness effective conductivities, as functions of the interfacial resistance, as well as thermal conductivities of bunches, $k_{b, e f f}^{n}$, are plotted in Fig. 5. The best fitted resistance, for assumed parameters, is close to $6 \cdot 10^{-}$ ${ }^{6} \mathrm{~m}^{2} \mathrm{~K} / \mathrm{W}$ (Fig. 5).

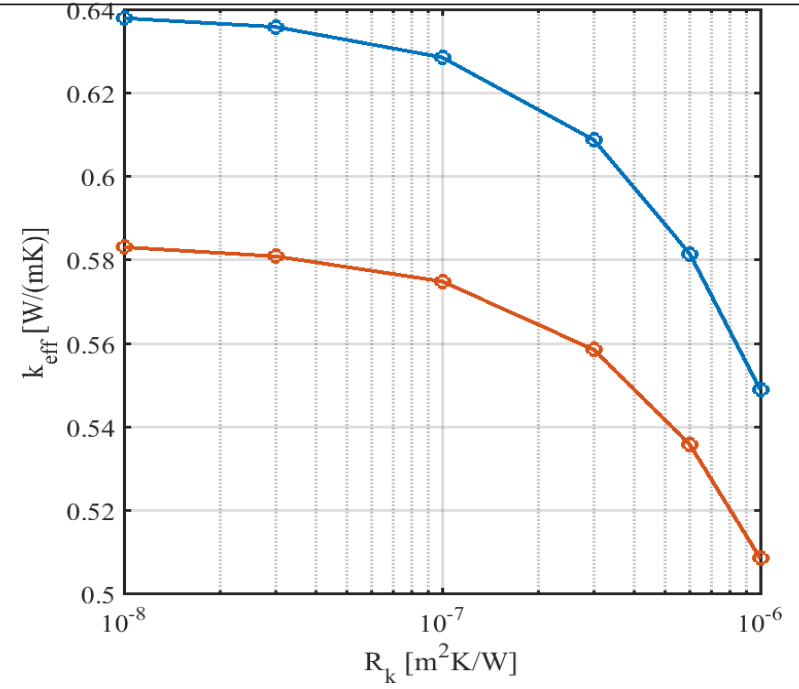

Fig. 5. Effective thermal conductivity of bunch of fibers infiltrated with epoxy (blue line) and predicted numerically conductivity of the composite in the $\mathrm{Z}$ direction (red line) as functions of thermal resistance.

Temperature distributions in the two crossections of the domain, perpendicular to the $\mathrm{Y}$ axis, crossing the half and the one-fourth of the domain width and on two side surfaces are presented in Fig. 6. The best fitted thermal resistance was adopted here. The temperature distribution in the crosssections is only slightly disturbed with the presence of epoxy resin, due to high fractions of bunches and immediate contact of bunches.

The impact of the void fraction of entrapped gas in the epoxy resin, present outside and inside bunches, on the overall effective thermal conductivity of the composite in direction normal to tows is next examined (Fig. 7).

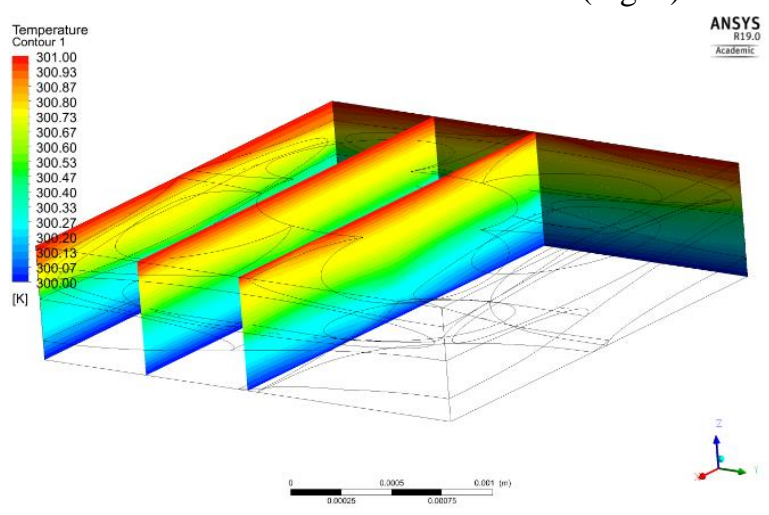

Fig. 6. Temperature map on two cross-sections of the domain and on two side walls.

Both numerical and experimental outcomes show the conductivity decreases with increasing volume fraction of voids. Numerical results underestimate the changes of conductivity. This can be induced by a change of carbon volume fraction as a result of higher compression forces introduced to the composite surface resulted from higher vacuum applied during manufacturing process. This was 
resulted in change of carbon fibre to matrix ratio which wasn't taken into consideration at numerical computations. However REV geometry was based on the microstructure of composite manufactured at the lower vacuum level $(90 \mathrm{kPa}$ below atmospheric preassure), which possessed the lower void volume fraction. Hence discrepancy of results in that case is minor.

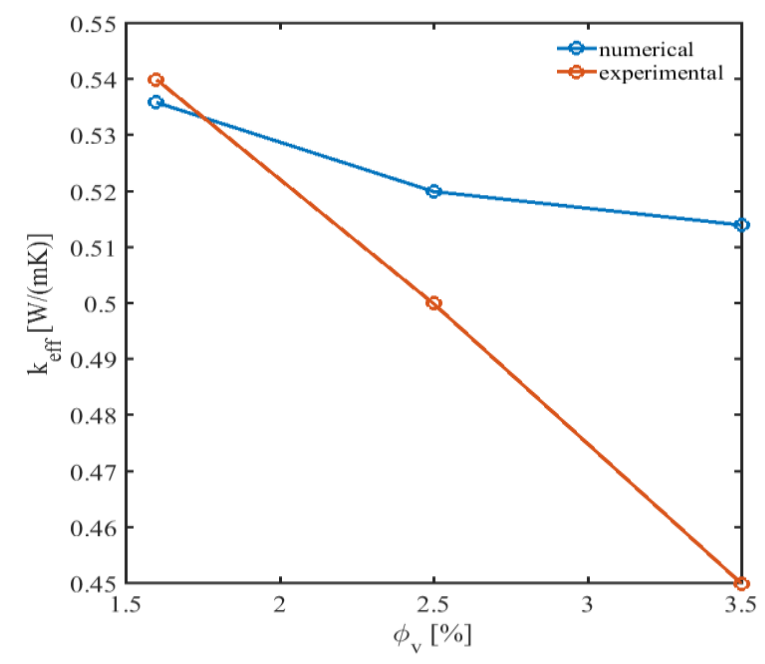

Fig. 7. Comparison of effective thermal conductivity in direction normal to yarns determined experimentally and predicted with numerical simulation.

\section{Conclusions}

In the proposed paper the computational model of heat transfer across the complex carbon fibre-epoxy composite, is proposed. The three dimensional geometry reflects the mesostructure of the composite components. The non-uniform micro-structure of warps, yarns and matrix material including anisotropic thermal conductivity of fibers, voids present in epoxy resin, and interfacial resistances on the surfaces of fibres is taken into account. The effective meso-scale thermal conductivities are determined with analitical formulae and next applied in the numerical meso-scale model. The interfacial resistance is estimated on the basis of simulations results, which assures the best fitting of the calculated throughthickness effective thermal conductivity with the determined experimentally one. For that determined resistance, the effect of void fractions in the epoxy resin on overall throughthickness thermal conductivity is investigated numerically. Numerical simulations underestimate this relation. However this could be caused by slight difference between modelled microstructure and the real one. It is exept composite manufactured at the lower vacuum level which, herein, was a benchmark for REV geometry development. Hence both experimentaly and computational results in this case are close. This slight alteration should be take into consideration in further works.

\section{References}

1. A. Boczkowska and G. Krzesiński, Kompozyty i techniki ich wytwarzania, OWPW (2016).

2. S.H. Goodman, H. Dodiuk-Kenig, Handbook of thermosets plastics, Wiliam Andrew (2014).

3. SAE International, Composites material handbook Vol. 2, CMH-17 (2016).

4. P. Czarnocki, A. Boczkowska, W. Frączek, P. Chabera, et al., , SAE Int. J. Mater. Manf., vol. 11, 2, (2018).

5. J.C. Maxwell-Garnett, Philos. Trans. Roy. Soc. London Ser. A, vol. 203, 385 (1904).

6. H. Fricke, J.Phys. Chem., vol. 57, 9, 934 (1953).

7. D.P.H. Hasselman, L.F. Johnson, J. Compos. Mater., vol. 21 6, 508 (1987).

8. D. Burggeman, Ann. Phys., vol. 24, 7, 636 (1935).

9. A.G. Every, Y. Tzou, D.P.H. Hasselman, R. Raj, Acta Metall. Mater., vol. 40, 1, 123-129 (1992).

10. R. Landauer, J. Appl. Phys., vol. 23 7, 779 (1952).

11. Z. Hashin, S. Shtrikman, J. Appl. Phys., vol. 33, 10, 3125 (1962).

12. T. Mori, K. Tanaka, Acta Metall., vol. 21 ,5, 571 (1973).

13. Y. Benveniste, J. Appl. Phys., vol. 61, 8, 2840 (1987).

14. Y. Agari, M. Tanaka, S. Nagai, T. Uno, J. Appl. Polym. Sci., vol. 34 , 4, 1429 (1987).

15. S. Zhai, P. Zhang, Y. Xian, J. Zeng, B. Shi, Int. J. Heat Mass Transf., vol. 117, 358 (2018).

16. Z. Han, A. Fina, Prog. Polym. Sci., vol. 36, 914 (2011).

17. J. X. Wei, "Report: Carbon fiber thermal conductivity measurement and analysis", Beijing Institute Mater. Sci. Technol. Div. (1989)

18. I. Hatta, T. Yamane, S. Katayama, M. Todoki, J. Wide Bandgap Mater., vol. 7, 294 (2000).

19. S. W. Beckwith, Composites reinforcement fibers : III - The carbon and graphite families (Part B), in SAMPE 49th International Symposium and Exhibition, vol. 47, 44 (2011).

20. N. Hu, S. W. Chiang, J. Yi, X. Li, J. Li, H. Du, C. Xu, Y. He, B. Li, F. Kang, Compos. Sci. Technol., vol. 110, 69 (2015).

21. K. Bui, B.P. Grady, D.V. Papavassiliou, Chem. Phys. Lett., vol. 508, 248 (2011).

22. T. Wejrzanowski, M. Lewandowska, K.J. Kurzydłowski, Image Anal.Stereol., vol. 29, 1 (2010).

23. T. Wejrzanowski, W.L. Spychalski, K. Rożniatowski, K.J. Kurzydłowski, Int. J. Appl. Math. Comput. Sci., vol. 18, 33 (2008).

24. J.C. Maxwell, A Treatise on Electricity and Magnetism. 3rd ed., chapter 9, 1, New York, Dover;(1954).

25. K. Pietrak, T.S. Wiśniewski,, Journal of Power Technologies, vol. 95,14 (2015).

26. A. Dasgupta, R.K. Agarwal, S.M. Bhandarkar, Compos. Sci. Technol., vol. 56, 209 (1996).

27. Y.S. Song, J.R. Youn, Carbon, vol. 44, 710 (2006) 